\section{INTERPRETATION OF NEGATIVE LABORATORY FINDINGS IN SYPHILIS}

With SPECIAL REFERENCE TO TREATMENT *

ALBERT E. STERNE, A.B., M.D.

Professor of Nervous and Mental Diseases, Indiana University School of Medicine

INDIANAPOLIS

We begin the consideration of this subject with several postulates:

1. A positive Wassermann reaction in the temperate zone spells syphilis, and only syphilis.

2. A negative or mild degree Wassermann reaction cloes not, by any means, indicate the absence of syphiiis.

3. Syphilis of the central nervous system is only part of general constitutional syphilis; it never occurs as a real entity and, furthermore, the nervous systcm is invaded early and not late, as is generally supposed.

4. It is absolutely necessary, especially in doubtful cases, to correlate laboratory and clinical data. The Wassermann and other serologic tests are mercly symptoms, which, like other physical signs, may or may not be present. They are extremely valuable, but not as determining as the objective clinical syndrone, notably when not definitely manifest. The laboratory is not a short cut to diagnosis.

5. Many laboratorians, while they may be excellent technicians, are incapable of determining the exact interpretation of the serologic reaction in debatable or doubtful cases. This decision rests with the clinician.

6 . Even with a positive Wassermann reaction a given syndrome in a known syphilitic patient is not always due to syphilis.

Each of these postulates requires some elucidation and comment.

Postulate 1.-A positive Wassemann reaction in the temperate zone denotes syphilis only.

Admitting that certain exceptions to the above given rule exist, and that a positive Wassermann reaction of moderate, or even high degree, occurs in the temperate zone, in conditions other than syphilis, such as pronounced malaria and other plasmodial affections, and in conditions of tropical disease migrate into the temperate zone, and again admitting that minor degrees of a positive Wassermann reaction may occur in certain other debatable conditions, nevertheless, as a routine dictum, we may safcly assert that a positive IVassermann reaction spells syphilis and syphilis only. The exceptional conditions differ so markedly clinically, that there should be no difficulty in ruling between them.

Recently it has been asserted that a positive bloodserum Wassermann reaction occurs in tuberculosis. It were well to bear in mind that there is no antagonism between syphilis and tuberculosis. The two may occur coincidentally in the same subject; they often do, in fact. Spinal fluid research in tuberculosis has, I believe, not been sufficiently carried on to decicle whether the alleged blood serum claim can be upheld.

Postulate 2.-A negatiz' Wassermann reaction does not indicate absence of syphilis. * Read before the Section on Nervous and Mental Diseases at the
Sixty-Ninth Anntial Session of the American Medical Association, Chicago, June, 1918 .
The negative phase requires careful analysis, and there are several different aspects involved in the negative side which require consideration. Before entering on their discussion, it will be well to establish clearly in our minds, certain facts in respect to syphilis of the nervous system, some of which, at least, are not generally recognized. This involves, in a general way, a discussion of Postulates 3, 4 and 5.

Postulates 3, 4 and 5.-The central nervous system is never, alone, involved in this disease; the other body tissues and organs are affected by it, inasmuch as syphilis is always a constitutional affection. Clinically, the symptomatology from the side of the nervous system may preponderate, and so far dominate, as to force the evidence of participation of other, even important, organs, into the background. However, the reverse is more likely to prove true, and we are much more likely to fail to realize the intrinsic participation of the central nervous system in the ordinary case of syphilis, because no signs of organic involvement of the brain or cord are manifested.

As a matter of fact, the central nervous system is involved, intrinsically, in every case of syphilis and, furthermore, it is involved early in the course of the disease and not late, as is commonly supposed. It is true that lesions, deep within the tissue of the neural system, are apt to be late manifestations, though not infrequently severe organic lesions of brain and cord occur within a few weeks of the initial infection. The syphilotoxins of the so-called secondary stage, however, affect the nervous system along with all the rest of the body organs, and it is at this period, especially, that a recognition of this fact by the physician may prove of the utmost service to the patient. Examination of the spinal fluid along with the examination of the blood, in the early stages of the disease, is far more important than in the later stages, but such examination is not ordinarily made.

In the syphilotoxic or so-called secondary stage, we are prone to encounter the characteristic evidence of toxemia in the mucoid and skin eruptions (which are in every sense analogous to the mucoid and skin eruptions of the infectious exanthems) associated with headache and often vertigo; the latter symptoms are strict indications of involvement of the nervous system and their occurence calls for investigation of the spinal fluid, as well as the blood. If there is any virtue or value in intraspinal medication, and I am not yet fully convinced that intramedullary injections possess unquestionable advantage, it should be applied at this stage, instead of, as now, waiting for the development of organic involvement of brain and cord, usually several years after the primary effect, when even intensive treatment is apt to fail. The most favorable side of our modern intensive specific therapy lies in the prevention of the disastrous late lesions of the nervous system, rather than in any attempt to arrest or to cure them after they have once become established. However, when, late or early, lesions of the brain and cord become manifest, whether of focal or diffuse type, we must again remember that the pathology affects the entire system. Tabes and other tract affections of the cord, transverse and diffuse myelitis, paresis and taboparesis are all clinical types of neural syphilis, but are merely part of general constitutional syphilis.

Years ago, prior to our present laboratory methods, good clinicians and, sometimes, mediocre ones, were perfectly capable of diagnosing syphilis in all of its 
aspects and relied entirely on their knowledge and clinical experience to arrive at definite conclusions. Today, they continue to rely on their keen diagnostic sense, but wisely substantiate their clinical conclusions by every laboratory test of value, though they are not absolutely controlled, or guided, by the laboratory data, which they recognize only as a part of thorough physical examination and not as a short cut to diagnosis. Unfortunately the reverse is apt to be true among busy general practitioners or so-called specialists, who either have not the ability, or the sense of deep personal responsibility in diagnosis, and they are inclined blindly to accept the verdict of the laboratory and be guided by it, without taking into consideration the clinical evidence, either in the affirmative or negative. The general run of the profession has not yet grasped the fact that all laboratory tests are relative and not absolute in value.

This is an age of strict scientific research, as it should be, particularly in medicine and surgery, and it becomes all the more necessary, on that account, to scrutinize carefully the reliability of the source of information on which good clinicians await only corroborative evidence and poor or careless clinicians place their boundless faith. As a general thing, the relationship between laboratorian and clinician is apt to be entirely too loose; this is particularly true as respects the common run of the profession and the large commercial laboratories, often far distant from the physician who sends in his material for analysis.

The careful diagnostician assures himself, to the largest degree, of the capability of the man to whom he entrusts his laboratory work, and after convincing himself of the thoroughness, technical ability and absolute interpretative reliability of the laboratorian, works with the latter in complete harmony and teamplay, consults with him in every sense, debating pro and con the clinical and laboratory evidence in any doubtful case. There is here the much-to-be-desired and necessary cooperation, essential to completeness of diagnosis and safety of the patient, and this is manifestly impossible of attainment in the strictly commercial laboratories, very few of which are reliable.

The first important essential, therefore, is to assure oneself of the absolute technical and interpretative reliability of the serologist or of the laboratory to whom our serums are submitted.

Now how shall we analyze his report? What does a positive Wassermann reaction denote? Does it signify that the disease itself is active or that the physical system is conducting an active fight against the malady? Does a negative Wassermann reaction indicate no syphilis or that the spirochetes are dormant and the body resistance supreme for the moment? Does a positive +++ , for instance, signify a major disease activity and minor + resistance or practically the reverse? Furthermore, who shall decide that a + or even $\mathbf{a}++$ represents a positive Wassermann?

At present, we construe a positive $(++$ or greater) report merely into necessity for specific treatment; and that is, after all, the most important, outstanding factor from the patient's point of view.

What then, shall we, as physicians do, in the face of a negative laboratory dictum, especially in a case of debatable type, either with or without ascertainable history of previous infection.

May we rest secure and say to the patient, "No syphilis, no treatment"?
It is an extremely important matter, both to patient and medical adviser, to determine this question. On the one hand, we might subject a nonsyphilitic subject to time-taking, very expensive treatment, such as would be indicated were a positive Wassermann reaction present. On the other, we would seriously err in counseling no specific treatment, when the patient in reality was suffering with syphilis and required intensive therapy.

In my experience, it has been far more frequent to mistake a negative or mild degree Wassermann reaction as an indication for no treatment.

Repeatedly, I have seen patients who had been advised, to their great, sometimes irreparable, damage, that antisyphilitic treatment was unnecessary, by physicians of presumed excellent standing, purely on the strength of a negative serum Wassermann reaction most frequently when no spinal fluid examination had been made. When, sometimes months, sometimes years, later, these same patients came into my care, there was manifestly distinct, clinically recognizable, syphilis of the nervous system, which might have been prevented had proper treatment been instituted in the face of a negative Wassermann reaction years before.

It is claimed by many laboratories and syphilographers that a serum Wassermann reaction may be provoked in a suspicious case by a small intravenous dose of arsphenamin twenty-four hours previously given, or by moderate doses of iodids for a period of from two to three weeks. This provocative possibility is denied by many, but, at present, it would appear safest in the interests of the patient, and of diagnosis, to subject the suspect to such measures as might insure a more definite laboratory finding. Here a note of warning may well be sounded. Undoubtedly, at times, indiscreet or tactless words by the physician affect the psychology of certain persons deleteriously, so that a word of caution may not be out of place. Unless the doctor is reasonably certain that syphilis is really in play, he should refrain from alarming any patient. The physician should study the individual mental attitude of every person consulting him and be certain that his advice to the patient be not misconstrtted. We talk too glibly about the Wassermann test.

The only safe guide in doubtful cases is to remember that all laboratory tests, whether of blood or spinal fluid, are merely symptoms, which like any other symptom or group of symptoms, may at times be absent. Like most cardinal signs in any disease, the positive Wassermann reaction will usually be found present in the vast majority of patients with active syphilis, but is frequently absent or only faintly indicated, even when the disease is manifestly in play.

That this is true is clearly substantiated by the reaction of the blood serum-less frequently the cerebrospinal fluid-in concrete instances, as shown by the Wassermann reaction before, during and after treatment, intravenously, alone, or in combination with intramedullary injections.

It is a common experience to find the Wassermann reaction varying in degree in any given case or group of cases. Often it is reduced to nil under the influence of arsphenamin, to again become active while yet the patient is under intense therapy. It is this very fact which renders it difficult to determine just when the case has been sufficiently treated. The mere reduction in strength of a one-time positive Wassermann reaction should not alone constitute a criterion of cure. 
The clinical syndrome, otherwise, must improve pari pass with the serologic findings. If this be not the case, then little or no headway against the spirochetes can be counted on.

If the clinical syndrome in specific cases be favorably affected, there is good reason to believe that the patient is deriving benefit from treatment, even though the laboratory findings fail to vary proportionately. $\mathrm{Up}_{\mathrm{p}}$ to date, however, the laboratory data form our best corroborative index as to the apparent security of the patient from probable reactivation.

It should be borne in mind that serum reactions are much more liable to vary than do the spinal fluid tests. The latter appear the more trustworthy, not only because of their greater stability, but also by reason of their multiplicity and, therefore, the possibility of checking up and correlating the other tests-leukocyte count, globulin increase and colloidal gold test-with the Wassermann.

The blood serum Wassermann test is of great diagnostic value, but of questionable prognostic worth. The spinal fluid tests are not alone diagnostic in character, but assume both differential and prognostic virtue. This is notably true of the Lange (colloidal gold) color curve. By it, when accurately done, we are enabled to determine with some assurance of ccrtainty, the character of the pathologic process transpiring in brain and cord, differentiating between inflammatory encephalitic-meningeal affections and probable degenerative tissue changes. True, this is not absolutely certain, but it forms a fairly reliable working index differentially and as to effects of treatment, and up to a certain point, as to whether treatment would be effective enough to warrant trial.

Where clear-cut degeneration of brain cells, as in true paresis, or cord tract and transverse lesions, tabosyphilis and myelitis syphilitica, is manifest, the effect of treatment is so questionable that one should gravely consider matters of time and expense among those who can ill afford to make financial sacrifices. Unfortunately, no matter how expert the clinician, we cannot with positiveness assert how much, in a given neurologic syphilitic syndrome, depends on inflammation and how much on degeneration. The experience in the past few years of intensive therapy, both with and without conjoined intraspinal treatment, should influence us always to give the patient the benefit of at least a trial, unless the case is so far advanced as to be apparently hopeless, by all standards of large experience, so that monetary sacrifice would be unjustified.

Postulate 6.-Even with a positize Wassermam reaction, a gia'en syndrome in a know'n syphititic may not be due to syphilis.

Not infrequently we find syphilitics suffering from nonsyphilitic affections, and it often becomes a difficult problem to solve therapeutically, Quite naturally the physician would be prone to conclude that such patients require intensive specific measures, and very likely many of them do, but the complex against which efforts are directed fails to yield. If this transpire, the doctor should not persist in the opinion that syphilis is responsible, but should direct his research otherwise. He is, however, always erring on the side of safety to bar out syphilis by adequate therapeutic attempt.

Another point to remember is that extraneous, often accidental, factors, not infrequently start a hitherto latent syphilis into activity. This, in my experience, has been particularly true in traumatisms to the head and back, to sun and heat stroke and to acute infections, such as grip, which are peculiarly liable to affect the nervous system. At the present time, too, we should not lose sight of the activating influence of war injuries and of war strain.

A final and extremely important phase of this entire subject is presented by the researches of Warthin, ${ }^{1}$ who has demonstrated spirochetes, postmortem, in practically every body-organ and tissue in many persons, who, intra vitam, had repeatedly given negative serologic returns. Are we to infer from Warthin's work that all our laboratory tests, and even our intensive antisyphilitic treatment, are worthless?

Unless I quite misunderstand Warthin's attitude, he has concluded that neither the Wassermann test nor any other laboratory test, as yet devised, has proved in any way trustworthy, believing that demonstrable spirochetosis exists too often to escape detection by a really valid test.

I, in common with most clinicians here and abroad, have faith in the Wassermann reaction, and other associated blood and spinal fluid reactions, always, however, weighed thoroughly in the clinical balance, but wish to emphasize the great value of Warthin's; revelations in not too rashly coming to the verdict of negative serum reaction, negative syphilis.

Spirochetes in organs and tissues after death, of course, spell syphilis at some period of life, but it remains yet to be proved that these organisms, morphologically intact, may not rest indefinitely in the tisstles, fixed and latent, without clinically active complexes in sequence. Warthin's work again serves well to emphasize the necessity for a test more reliable even than the Wassermann.

\section{SUM MARY}

1. A positive Wassermann reaction of blood, and especially of spinal fluid, spells syphilis and syphilis only.

2. A negative serum Wassermann reaction and even a negative spinal flutd Wassermann reaction does not strictly indicate the absence of syphilis.

3. Laboratory tests are merely clinical signs, which at times may be present or absent, like other symptoms; spinal fluid tests should always be made together with blood serum tests.

4. Correct interpretation can be made only by weighing carefully the clinical evidence, with the serologic reports, especially when the litter are negative. This cannot be too emphatically emphasized.

5. Laboratorian and clinician should hold far closer scientific relationship than is now usual. They should be mutually helpful.

6 . The laboratory findings should fit into the clinical syndrome and not vice versa.

7. And unless there be wise, deliberative interpretation of laboratory data, especially negative data, in neurologic and other cases, serious errors in diagnosis and treatment will continue.

Note.-Since the foregoing was written, a paper by Drs. Larkin, Levy and Fordyce (The Journal, June 1, 1918, p. 1589) refers to this subject in most timely way. The reader is advised to study this article. Its careful perusal will serve to emphasize what has herein been stated.

1. Warthin, A. S.: The Persistence of Active Lesions and Sniro. chetes in the Tissues of Clinically Inactive or "Cured" Syphilis, 


\section{ABSTRACT OF DISCUSSION}

DR, C. R. BALL, St. Paul: This is a subject that I have been interested in for a long time. I presented before this section the first paper on the four reactions. Since that time, in my own laboratory and clinical work, I have very carefully followed these reactions almost daily. As illustrative of one point I will relate the case of a patient, a man of 30 , who had a fairly good history of having had a chancre some six years ago. When I first saw him he had not one single hair on his head; his eyebrows were gone, and his finger and toe nails had a marked degree of onychia. $\mathrm{He}$ stated that he had a negative serum Wassermann. I made spinal fuid tests and found the reaction serobiologically to be absolutely negative; yet the clinical symptoms would have caused one to conclude that this was a case of syphilis. He responded well to specific treatment, and in six months he had enough hair on the back of his head to require a hair-cut. So far as a cure is concerned, the reactions may be of value in determining whether or not recovery bas taken place, but when we consider the patient just described we must conclude that it is not a reliable criterion.

On the other hand, we are familiar with that type of patients who come to us with positive reactions, but who look the picture of health and present no clinical symptoms. Three years ago a man came to me just after attending the funeral of his brother who had died of paresis. He had another brother suffering from locomotor ataxia, and, remembering that he had contracted syphilis some fifteen to twenty years before, he began to worry over himself. Physically and clinically he was well. He had a negative serum Wassermann, but his blood reacted typically to what we term the characteristic paretic reactions, the colloidal gold, the globulin, the cell count and the Wassermann, there being $a+++$ or ++++ Wassermann with the minimum amount of spinal fluid: A week or two ago $I$ saw this man again and he was free from any symptoms and apparently in perfect condition. The longer I deal with these reactions the more I feel that their usefulness at present lies in what we get out of them in the way of diagnosis. I have been much interested in the treatment of these conditions. It seems to me that we must compare syphilis with tuberculosis. Dr. Riggs and Dr. Sterne have pointed out that syphilis is not an isolated disease of the nervous system, but that it is a constitutional disease, and that the nervous system is involved at the same time that the rest of the body is involved. Years ago we believed that to give the tuberculous patient large doses of creosote would kill the tubercle bacillus, and we have been passing through much that same phase with reference to methods of intensive treatment of syphilis.

Dr. Julius Grinker, Chicago: Most of Dr. Sterne's statements can le subscribed to because every one of us has had simliar experience with the Wassermann tesis. One point on which Dr. Sterne did not lay stress, and which I wish to emphasize, is that we occasionally get a negative Wassermann in a very advanced case of syphilitic infection. Not alone does this occur occasionally in paresis, but most frequently in advanced cases of tabes. Some years ago, for the first time in Chicago, I injected five patients intraspinally, following the Swift-Ellis treatment. One case was diagnosed as tabes from the symptoms present, but it was not an advanced case. I presented the patient before the medical society as one who had improved under Swift-Ellis treatment-that this treatment had done a great deal of good, as the Wassermann was made negative after being strongly positive. This man left the hospital, and returned a year later with a most advanced type of tabes, was extremely ataxic, had Argyll Robertson pupil, intense pain, sphincter incontinence, etc. But because of the negative Wassermann test and other signs that were negative we waited for corroborative evidence. We took specimens of spinal fluid and blood, and the tests made were absolutely negative, yet the man was advanced to a very bad case of tabes and could not be cured by any treatment.

I have tried the Swift-Ellis treatment in paresis, and in one case I was extremely proud of the results. The patient was very violent, and for this reason had been thrown out of three different rest cures and one hospital. We finally succeeded in quieting his mental symptoms, he became rational and is today going about and is able to enter my office without difficulty. That patient, though he has improved mentally, has developed the physical signs more markedly after intensive treatment than before, showing that the disease relentlessly progresses just the same, causing its physical signs. Whereas he had no Argyll Robertson, although on both sides he had a sluggish pupillary light reaction, he now has a complete Argyll Robertson. How long he will live I do not know, but mentally he was benefited by the Swift-Ellis treatment, and some other patients who I am now treating, both tabetics and paretics, have shown remarkable improvement. I am still looking for the cures in paresis and tabes.

Dr. Walter Timme, New York: In this connection I wish to mention a point brought out by Dr. Sterne, namely: That in the temperate zones a positive Wassermann practically means syphilis. In the last two or three years I have had an opportunity to examine many cases of lead intoxication, largely those without peripheral lesions, affecting the central nervous system and giving the symptoms of lead encephalitis; and these cases invariably give a positive Wassermann. Sir Thomas Oliver reports the same condition as being present in the lead workers in English mines, and similar reports have come from Paris. In New York, besides our own findings, Cyrus Field has reported several such cases. Other cases have been reported from South America, with the statement that clinical syphilis could be excluded. One particular case that I have in mind may throw some light on the subject: A boy, 14 years of age, came to me suffering with what looked like juvenile paresis, and his condition had followed more or less quickly his occupation in a painter's shop, his work consisting of sandpapering off of the paint preparatory to repainting. Within a few weeks after entering on that occupation he began to show the signs of loss of memory, intense headaches, and speech disturbances, such as occur in paresis. The other members of the family were examined, and all gave a negative history and negative findings as to syphilis, yet this boy showed a positive Wassermann reaction in the blood and cerebrospinal fluid. He improved very markedly under treatment for lead intoxication. $\mathrm{He}$ was given no antisyphilitic treatment whatsoever, and left, not cured, but very much improved. That is a case in which syphilis had almost to be excluded, and yet it is a case corroborative of the findings of other clinicians throughout the world.

Dr. Elmer E. Southard, Boston: In respect to what Dr. Grinker said, I would ask the names of the distinguished psychiatrists and alienists who have cured paresis. I am not aware of any such cures, by men distinguished or otherwise. They may paint the topic in a rosy hue and try to encourage one, but it looks to me as if it was going far to sperik of a cure.

I wonld like to ask Dr. Sterne what his medicolegal answer would be in a case such as that afforded by his title. I want to cite a supposititious case. A man who has been awarded money and then fallen into a paretic state, as he thought, with amnesia, with stupor, with symptoms that were undoubtedly paretic, clinically speaking, was then treated rather successfully so that all his laboratory signs, which had been positive, became negative; no mental symptoms are present and he has nothing left but the Argyll Robertson pupil. He now is earning $\$ 25$ a week, but if he could get into a certain trade he would be able to make much more money. He came to our hospital for a letter of approval. What is the answer to this question?

Dr. Andrew L. Skoog, Kansas City: Dr. Sterne has called attention to several valuable points. Regarding the opinions of general practitioners concerning the value of laboratory findings in these cases, it has been my observation that their views are quite variable. You will find one group of practitioners who throw aside the laboratory findings entirely; another group of men, as indicated here, rely on the laboratory findings alone. As Dr. Sterne has pointed out, that is absurd, in that not infrequently we get negative findings in both blood and spinal fluid, whereas there are undoubtedly 
positive evidences of syphilitic disease. There is no doubt but that the spinal fluid analysis is of more importance than the blood analysis for syphilis of the central nervous system. When we analyze the seat of the disease we must bear in mind that syphilis is a disease that is attracted to the blood vessels and neighboring tissues. The pia mater is intimately associated with the spaces which furnish the spinal fluid; therefore it is quite readily conceded that in syphilis bodies are thrown out in the spinal fluid which will give us our findings and that there will be consequent leukocytosis and other evidences of the disease.

Dr. Frank P. Nokbury, Springfield, Ill.: I would like to report a case. A woman, married, had been using the lead acetate solution with the result of lead poisoning and development of symptoms of syphilopsychosis type. The laboratory findings were those of a positive Wassermann. I supposed we had to deal with syphilis in connection with lead poisoning, but the clinical history of lead poisoning was definite so far as the findings were concerned. Recovery was effected under antilead treatment. So far as other findings are concerned, I do not know anything about that, as the patient has disappeared from observation.

In regard to what Dr. Ball has said as to the diagnostic value of the Wassermann, $m y$ associate and $I$ have taken the Wassermann in all cases, and in several cases, especially in women whose social status was undoubted, we have noted what might be called a neurasthenic condition, and yet it is not a definite neurasthenic condition eitiser; it is more of a restless irritability, with inability to get along at home in respect to the social environment and disturbed in many ways. In several of those cases we have found the positive Wassermann, leading us to believe that that may have and does have to do with the condition present. Under appropriate treatment these patients improve and many have returned home. I do not know what the findings will be later. One case under observation has developed paresis, so this was the preparetic irritation which we saw.

Dr. Francis X. Dircum, Philadelphia: I am very glad to see the emphasis lairl on the clinical lindings. When the Wassermann reaction was lirst brought before the profession there was a stampede to the laboratory, but we are now coming back to a deeper consideration of the clinical symptoms. I want to call attention to a point connected with tension, and that is the great value of spinal drainage. I do not think this point is fully appreciated. It is not sufficient to withdraw five or six cubic centimeters; you must withdraw sixty or seventy cubic centimeters. In carrying out this procedure we have not had a single mishap, and we have made many spinal drainages. It seems to me the benefit that accrues may be explained as follows: In the first place, in tabes and paresis both the spinal fluid and the cord are under great pressure, and under these circumstances the amount of fluid entering the cord must be diminished. If the pressure is reduced the cord must become hyperemic, and perhaps some of the astounding results we sometimes get in tabes from repeated drainage, every ten days or two weeks, even in the absence of other treatment, is explainerl by this. Drainage, absolute and complete, is a point of great value.

Dr. G. A. Moleren, Denver: I am not willing to take the Wassermann reaction from the sphere of contributory evidence. I think it is going to remain there for some time to come, and we must be guided chiefly by the clinical symptoms. I also want to bear testimony to the extreme value of drainage of the spinal cord. However, I must say that even though I had discussed the matter with Dr. Dercum, I felt some hesitation and fear, which I think we would all feel, on the first thought of draining every bit of fluid from the spinal canal. After Dr. Dercum had placed the matter on record I drained in quite a number of cases, and when I drained completely, every one of them would drop a cell count anywhere from 150 to 300 cells, after eight, ten or fifteen drainages, to something under ten. The principal point I wished to make, however, is with reference to the general evidences of infection of the nervous system and the treatment to be applied in accordance with that view. I do not think we are willing to take the position that every case of specific infection should be intraspinally treated because of fear of infection of the nervous system. We know that most of the cases of syphilis do not become neurosyphilitic. I do think the infection of the nervous system itself is nearly always indicated by the laboratory findings in the spinal fluid, and $I$ think we have a great many cases which show positive blood reactions and no evidence in the spinal fluid. I do not think we are ready to accept the views that have been expressed, especially by McIntosh, that the nervous system is sensitized in the secondary stage of syphilis and that we have the so-called psychic symptoms of an anaphylactic reaction. In other words, I am opposed to the view that the general infection of syphilis is necessarily an infection of the ncrvous system, although I am quite willing to accept the view that no syphilis, no paresis and no tabes.

Dr. Otto G. Freyermuth, San Francisco: Dr. Ball brought out the very important fact that one could have a latent syphilis without any laboratory findings, and he showed how he continued treatment with very marked contintous absence of laboratory findings. I would like to ask Dr. Ball if, after he had instituted the antisyphilitic treatment in the case he referred to, he took the laboratory findings then to determine whether or not there was any evidence of positive syphilis. I do not feel that we are justified in excluding our laboratory findings entirely, yet we should not place too much stress on them. But, if in the laboraiory tests of the spinal fluid we should find $a+++$ positive Wassermann, a high cell count, a positive globulin and a positive colloidal gold, I think there can be no question of syphilis in that particular case. But in the presence of a positive Wassermann alone, and only one or two plus, I would certainly hesitate before giving a positive diagnosis of syphilis. The statement has been made that under the antisyphilitic treatment and the Swift-Ellis drainage, certain mental manifestations had cleared. but that later on the tabetic manifestations were intensified. $\mathrm{My}$ experience in thiat respect is that the mental condition cleared up due to the releasing of the spinal fluid pressure, and, perliaps, the Swift-Ellis treatment had nothing whatever to do therapeutically with the mental conditions. My experience has been that drainage is a great necessity. The work done by Dr. Mehrtens of the Stanford Clinic would show how we can improve a great deal on the drainage by the modified Swift-Ellis treatment.

Dr. Tom B. Throckmorton, Des Moines: The statement marle by Dr. Dercum concerning the use of drainage of the lumbar sac in these cases is of sufficient importance to attest to the value of this method as an adjuvant in treatment. About three years ago Dr. Dercum told ne about this work, and since then I have used this method quite extensively in cases in which I could have patients confined either at home or in hospital service. In the vast majority of instances in which there were prodromes of threatened crises in talses, I have prevented or entirely alleviated those symptoms which are so annoying in cases of tabes with spinal root irritation, by using the drainage method.

Dr. Waltir F. Schaller, San Francisco: The work of Dr. Henry G. Mehrtens in the Stanford Clinic is based on the attempt to increase the permealility of the meninges or the choroid plexus to the passage of drugs administered intravenously. In order to accomplish this, an intraspinal injection of the patient's blood serum or horse sertm is made, and after a sufficient interval of time for the meningeal reaction to take place the drug is given by the ordinary intravenous route. By this method sodium iodid is found to pass into the cerebrospinal fluic. We are now using arsphentamia by the method which is in effect a reversal of the usual procedure by the Swift-Ellis treatment.

Dr. Joseph BYrNe, New York: It seems to me that we have struck the little snag that is so common in the diagnostics of medicine when we lose sight of the logical evaluation of things. Some people are keener in perception and logical evaluation of evidence than others. And it impresses me as very strange how much importance we attach sometimes to a negative finding. A negative finding must always be taken as just a negative finding. If we want to attach any impor- 
tance to it we must follow the corroborative method as in all reasoning by induction. We must not attach too much importance to negative conclusions based on one or two examinations. We may have a positive spinal fluid with no clinical signs; then, again, we may have a negative spinal fluid with positive clinical signs. We must always take what is primary and fundamental as far as diagnostics go, namely, the positive thing. Give that its valuation and then search for corroboration. Negative laboratory findings can never nullify positive clinical evidence Then, again, we have the problems that are raised by Dr. Timme's case. I do not believe, however, that Dr. Tinme has been able to exclude syphilis. Dr. Dercum spoke of positive clinical findings. We have of late been too prone to overlook the value of accurate, wellrecorded clinical studies. Certainly the men of fifty or sixty years ago were infinitely our superiors in the study and evaluation of clinical evidence. It is to be hoped that we will finally come back to the true evaluation of positive and negative findings, whether these come from the laboratory or from clinical observation.

Dr. Jostry M. Aikin, Omaha: The clinical findings are growing in importance. The laboratory findings are of great value as contributory evidence in diagnosis. But I believe that many men in general practice have given them a place of paramount importance. A case by way of illustration: A lady uncier 30 sent one of the surgeons in our city a telegram to be ready to operate on her at a certain hour in the forenoon in one of the hospitals. The surgeon met the patient, refused to operate, and asked me to see the case. It was a frank case, clinically speaking, of syphilis. It was saicl to be a case of gallstone trouble. Under antisyphilitic treatment we had splendid results. Examination of the blood and spinal fluid was macle by a very good laboratory man, and he said that it was not a case of syphilis. I presented the case to my students and they voted unanimously that the case was one of syphilis irrespective of what the laboratory findings showed.

Dr. Albirt E. Sterns, Indianapolis: Relative to Dr. Timme's case, while it is true that the laboratory findings in cases of encephalopathy of the lead type simulate those of syphilis, there is one distinctive feature and that is the basophilia which occurs in learl cases and not in syphilis, and that should bar out syphilis in these cases. I cannot answer Dr. Southard's question without seeing the man. I was glad to hear the emphasis accorded to what I tried to bring out. Papers read here are published in a general medical journal; we are not now just talking among ourselves, but what we say goes out to the profession as a whole. And there is no questioning the assertion that the general profession does not fully realize the fact that the lahoratory is being used as a short cut, not alone in the diagnosis and treatment of syphilis, but in many other diseases, pulmonary tuberculosis, for instance. It is a question for the clinician to decide whether be has a case of tuberculosis to deal with, not because there are tubercle bacilli in the sputum, but because the physical signs definitely point to such an affection. In. respect to the Wassermann test, I call attention to this fact: Since this paper was written a reply has been marle by Fordyce and his co-workers to Symonds' suggestions as to the value of the Wassermann reaction. Fordyce emphasizes the positive value of the Wassermann reaction, and his paper is extremely valuahle. Symonds' article would seem to indicate that the Wassermann test is valueless. Warthin, again, absolutely stands on the proposition that there is no valid test for syphilis. He simply asserts that he arrives at this conclusion, because spirochetes can be demonstrated after death in individuals giving negative laboratory tests during life. And this is true, but the presence of the spirochetes after death simply indicates that during the life of the individual he had acquired syphilis, and that is all. Spirochetes may remain dormant and fixed in the tissues for years and give no laboratory reaction, such as we know. I do not think a wise clinician would wholly rely on laboratory findings, neither would he take the point of view that there is no value to these tests. There is a swing of the pendulum in the opposite direction now. Many men have come to the view that there may be nothing in the Wassermann, and I think that is wrong. The Wassermann is without doubt an extremely valuable reaction and we should hold to it, but the Wassermann, as generally made, may be valueless. In the first place, a lot of people who cannot make them think they can make Wassermanns, and, in the second place, there is no standardization of technic for a Wassermann reaction, either of the blood or spinal fluid. We should have standardized methods, and antil we have these, a statement of the method employed should accompany the laboratory report to guide the clinician as to its comparative value. As to sending serums to a large commercial laboratory, we must know what condition the serums are in when they reach the laboratory. The men in charge want the $\$ 5$ or $\$ 10$, as the case may be, therefore they are not going to write back that the serum arrived in such condition they could not use it. There is nothing of reliability to be attached to a test made under such circumstances. With reference to Dr. Moleen's point of view, I said very emphatically that I was not convinced that intraspinal treatment had the value which is ascribed to it by some. After many years of use, I feel that I can subscribe to the intensive intravenous treatment associated with spinal drainage. Intravenous injection is the method which has given me by far the best results. I am getting away from intraspinal injections, but doing more and more spinal drainage together with intravenous intensive medication.

\section{VISCERAL SYMPTOMATOLOGY IN NERVOUS DISEASES}

GRAVE DANGERS OF MISINTERPRETATION AND OF UNNECESSARY SURGICAL INTERVENTION *

\section{FRANCIS X. DERCUM, M.D.} PHILADELPHIA

At the present day, owing to the development of the various specialties, a development which has gradually become more and more intensive, the proper values are not always assigned to the symptoms presented by a given case. A physician accustomed daily to observe his patients from a single point of view is naturally in danger of acquiring a narrowed horizon. This danger can only be avoided if he keep in touch in greater or less degree with the other departments of medicine, especially with the great field of neurology. The specialist naturally has his attention attracted to some one organ or group of organs; perhaps it is the stomach, the heart, the genito-urinary apparatus, or perhaps a special sense organ. It may be that the symptoms presented owe their origin exclusively to the local conditions, or it may be that the latter form but a part of a much larger symptom-group. To assign to a special symptom its proper value at once becomes a matter of great importance. On the correct solution of this problem depends not only the diagnosis, but the subsequent treatment and very frequently the question of a surgical interference. I may add that in the field here presented crass differences of opinion have from time to time obtained, and that it is today a territory in which a veritable struggle is taking place; at times between the internist and the neurologist, but much more frequently between the internist or neurologist on the one hand and the surgeon on the other. In no department of medicine does this become more apparent than in neurology. Here, both in functional and organic nervous diseases, the affections may express themselves by visceral symp-

* Read before the Section on Nervnus and Mental Diseases at the Sixty-Ninth Annual Session of the American Medical Association. Chicago, June, 1918 . 